\title{
Tired heart and mute brain: handle with care
}

\author{
E. Koirala ${ }^{1}$, A. Chapagain ${ }^{2}$, L. Thapa ${ }^{3}$, M. Gautam ${ }^{4}$, R. Paudel ${ }^{3}$, B.R. Pokhrel ${ }^{3}$, PVS Rana $^{3}$ \\ ${ }^{1}$ Intern, ${ }^{2} \mathrm{MBBS}$, Final year, ${ }^{3}$ Department of Neurology, ${ }^{4}$ Department of Cardiology, College of Medical Sciences-Teaching \\ Hospital, Bharatpur, Nepal
}

\begin{abstract}
Managing a case of stroke is complex. Presence of variable sized brain infarct and heart failure is even more complex to manage. On top of this, presence of clot in the heart adds to confusion in medical management of stroke patients because of limited scientific evidences. With increasing number of stroke patients and improving diagnostic facility, it is interesting to find thrombus in ventricles of few of these patient, which otherwise were uncommon previously in our set up. We present a 64-year-old man, a case of ischemic stroke caused by left ventricular thrombus secondary to dilated cardiomyopathy and highlight the present recommendation to manage the condition.
\end{abstract}

Key words: Stroke, left ventricular thrombus, cardiomyopathy.

\section{Introduction}

Stroke is sudden onset of a neurologic deficit from a vascular mechanism which may be either ischemic or hemorrhagic. Amongst various etiologies, ischemic stroke is most often due to embolic occlusion of large cerebral vessels; source of emboli may be heart, aortic arch or a more proximal arterial lesion. We present a 64-year-old man, a case of ischemic stroke caused by left ventricular thrombus secondary to dilated cardiomyopathy and highlight the present recommendation to manage the condition.

\section{Case Report}

A 64-year-old man had presented to emergency department with sudden onset weakness of right half of body and slurring of speech of 4 hours duration. He had associated right sided facial weakness. He denied history of heart disease. He had untreated hypertension for last 5 months. He was also known to have Chronic Obstructive Pulmonary Disease and was a chronic alcoholic. He presented with GCS of 13/15and blood pressure of 120/80mmHg. Cardiovascular examination was normal. C-reactive protein was negative and other blood investigations including lipid profile was normal. Computerized Tomography (CT) of head showed infarct in left parietal region (Fig. 1). Electrocardiography (ECG) showed sinus tachycardia

Correspondences: Ms.E. Koirala

E-mail: frenkliza@hotmail.com and transthoracic echocardiography revealed global hypokinesia, severe left ventricular systolic dysfunction (LVEF 20\%), moderate MR, mild TR with dilated LA and LV. Thrombus was noted in apex of left ventricle (Fig. 2). He was managed with acute stroke protocol and he was discharged successfully on warfarin with Modified Rankin Scale of 2.

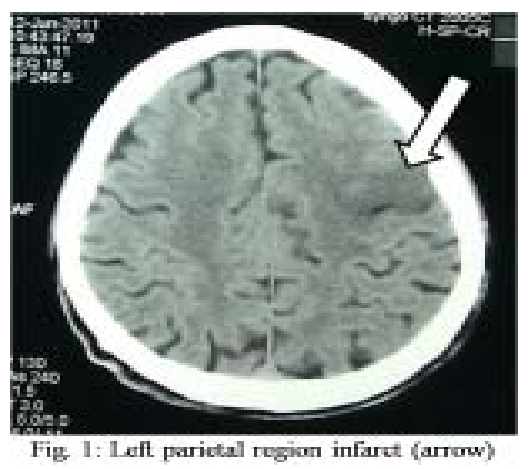


E. Koirala et al. Tired heart and mute brain: handle with care

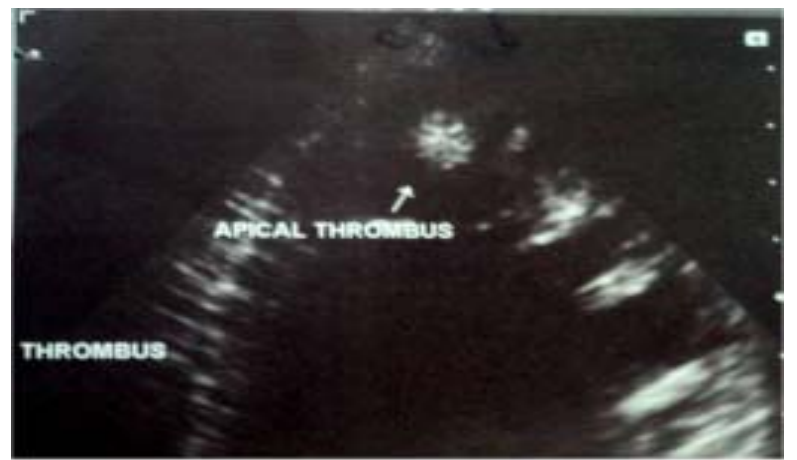

Fig. 2: Transthoracic echocardiography showing left ventricle apical thrombus

\section{Discussion}

Stroke is an increasing public health concern throughout the world; it is the second commonest cause of death and the leading cause of long-term disability worlwide. ${ }^{1}$ The incidence of cerebrovascular disease varies in different parts of the world. This group of disorders has become a vital importance in world health with increasing age of population. ${ }^{2}$

Amongst various important etiologies, cardioembolic source remains an important cause for ischaemic stroke. In general, about $20 \%$ of ischaemic strokes are caused by large vessel atherosclerosis (including the aortic arch, intracranial or extra-cranial large arteries), $20 \%$ by small vessel disease, $30 \%$ by cardioembolism, $5 \%$ by miscellaneous causes (e.g. carotid artery dissection), and $25 \%$ are of undetermined aetiology. $3,4,5$

Cardioembolic strokes are probably over-represented because finding of a potential source of embolus does not necessarily mean that a stroke is embolic. ${ }^{6}$ Atrial arrhythmias, congestive heart failure with low ejection fraction, and akinetic regions are most common sources of brain embolism. The incidence of stroke is inversely proportional to ejection fraction (EF) ${ }^{7,8}$

Conditions that affect endocardium and myocardium promote the formation of cardiac mural thrombi. Mural thrombus formation and embolism are commonest in dilated cardiomyopathy. In this group of patients, mural thrombi form mostly within the trabeculae carneae near the cardiac apex. ${ }^{9}$ Because of limited scientific evidences, managing patients with stroke with clot in the heart remains a big challenge. Treatment of patient with brain embolism is directed to open the occluded vessels by the use of drugs that lyse clots or use devices to extract thromboemboli. ${ }^{9}$

The decision to use heparin acutely to prevent the next thrombo-embolic event should depend on weighing the risk of acute re-embolization versus the risk of hemorrhage (eg. large infarct size) related to heparin therapy. Heparin does not lyse the existing thrombi although cardiac clots often disappear during heparin treatment. Acute heparinization in MI with mural thrombi is probably warranted but there is lack of evidence to support its use acutely in chronic congestive cardiac failure with mural thrombus. Direct thrombin inhibitors like dabigatran might change the method and risk of anticoagulation. ${ }^{9}$ Anticoagulant treatment is reasonable for chronic prophylactic treatment to prevent re-embolization in patients with cardiomyopathy with intraventricular thrombi but controversy exists in the use of warfarin in patients with low ejection fraction without thrombus.

Current recommendation for patients with ischaemic stroke or TIA who have dilated cardiomyopathy, either warfarin (INR of 2.0 to 3.0) or antiplatelet therapy may be considered for prevention of recurrent events (Class IIb, Level of Evidence C). ${ }^{10}$

To conclude, stroke as a result of ventricular thrombus secondary to dilated cardiomyopathy 
should be managed carefully considering the extent of cerebral ischemia, cardiac status, and present recommendation (although weak and of low level evidence).

\section{Learning points}

1. Murmur may not be appreciated clinically in patients with low ejection fraction.

2. Left ventricular thrombus in stroke patients should be treated acutely with heparin weighing the risk benefit ratio.

3. Oral anticoagulation may be better option for long term management to prevent re-embolization in dilated cardiomyopathy.

Acknowledgement: We would like to thank Mr. Surya Ghimire for his cooperation.

\section{References}

1. G.A. Donnan, M. Fisher, M. Macleod et al. Stroke. Lancet 2008; 10; 371(9624):1612-23.

2. J. Abraham, P. S. S. Rao, S. G. Inbaraj et al. An Epidemiological Study of Hemiplegia due to Stroke in South India. Stroke 1970; 1; 477-81.

3. J.M. MacKenzie. Are all cardio-embolic strokes embolic? An autopsy study of 100 consecutive acute ischaemic strokes. Cerebrovasc Dis 2000; 10: 289-92.
4. T. Moulin, L. Tatu, F. Vuillier, et al. Role of a stroke data bank in evaluating cerebral infarction subtypes: patterns and outcome of 1,776 consecutive patients from the Besancon stroke registry. Cerebrovasc Dis 2000; 10: 261-71.

5. G.W. Petty, R.D. Brown, J.P. Jr. Whisnant, et al. Ischaemic stroke subtypes. A population based study of incidence and risk factors. Stroke 1999; 30: 2513-6.

6. R.E. Kelley. Mechanism of in-hospital cerebral ischaemia. Stroke 1986; 17: 430-3.

7. M.A. Pfeffer, E. Braunwald, L.A. Moye. et al. Effect of captopril on mortality and morbidity in patients with left ventricular dysfunction after myocardial infarction: results of the survival and ventricular enlargement trial: the SAVE Investigators. N Engl J Med. 1992; 327:669-77.

8. E. Loh, M.S. Sutton, C.C. Wun, et al. Ventricular dysfunction and the risk of stroke after myocardial infarction. N Engl J Med. 1997; 336:251-7.

9. R. Louis. Caplan. Caplan's stroke: A clinical approach. $4^{\text {th }}$ edition. USA: Saunders; 2009:339.

10. L. Ralph. Sacco, Greg Albers, Karen Furie et al. AHA/ ASA Guidelines. Guidelines for Prevention of Stroke in Patients with Ischemic Stroke or Transient Ischemic Attack: A Statement for Healthcare Professionals from the American Heart Association/American Stroke Association Council on Stroke. Available at: http:// stroke.ahajournals.org/content/37/2/577.full. Accessed July 1, 2011 